Trinity University

Digital Commons @ Trinity

Psychology Faculty Research

Psychology Department

$7-2013$

\title{
Eating Disorder Prevention: Current Evidence-Base and Future Directions
}

\author{
Eric Stice \\ Carolyn Becker \\ Trinity University, cbecker@trinity.edu
}

S. Yokum

Follow this and additional works at: https://digitalcommons.trinity.edu/psych_faculty

Part of the Psychology Commons

Publication Details

International Journal of Eating Disorders

\section{Repository Citation}

Stice, E., Becker, C.B., \& Yokum, S. (2013). Eating disorder prevention: Current evidence-base and future directions. International Journal of Eating Disorders, 46(5), 478-485. doi: 10.1002/eat.22105

This Article is brought to you for free and open access by the Psychology Department at Digital Commons @ Trinity. It has been accepted for inclusion in Psychology Faculty Research by an authorized administrator of Digital Commons@ Trinity. For more information, please contact jcostanz@trinity.edu. 


\title{
Eating Disorder Prevention: Current Evidence-Base and Future Directions
}

\author{
Eric Stice, $\mathrm{PhD}^{1,{ }^{*}}$, Carolyn Black Becker, $\mathrm{PhD}^{2}$, and Sonja Yokum, $\mathrm{PhD}^{1}$ \\ ${ }^{1}$ Oregon Research Institute, Eugene, Oregon \\ ${ }^{2}$ Trinity University, San Antonio, Texas
}

\section{Abstract}

Objective-This narrative review sought to (a) characterize prevention programs that have produced reliable, reproducible, and clinically meaningful effects in efficacy trials, (b) discuss effectiveness trials that have tested whether prevention programs produce intervention effects under ecologically valid real-world conditions, (c) discuss dissemination efforts and research on dissemination, and (d) offer suggestions regarding directions for future research in this field.

\begin{abstract}
Conclusion-A literature revealed that 6 prevention programs have produced significant reductions in eating disorder symptoms through at least 6-month follow-up and that 2 have significantly reduced future eating disorder onset. Effectiveness trials indicate that 2 prevention programs have produced effects under ecologically valid conditions that are only slightly attenuated. Although there have been few dissemination efforts, evidence suggests that a community participatory approach is most effective. Lastly, it would be useful to develop programs that produce larger and more persistent reductions in eating disorder symptoms and eating disorder onset, focus more on effectiveness trials that confirm that prevention programs produce clinically meaningful effects under real-world conditions, conduct meditational, mechanisms of action, and moderator research that provides stronger support for the intervention theory of prevention programs, and investigate the optimal methods of disseminating and implementing evidence-based prevention programs.
\end{abstract}

\section{Keywords}

eating disorders; body dissatisfaction; prevention; effectiveness; dissemination

\section{Introduction}

Approximately $10-12 \%$ of young women in Western countries meet criteria for DSM-IV anorexia nervosa, bulimia nervosa, or eating disorder not otherwise specified (EDNOS), which includes sub-threshold eating disorders and partial eating disorders, such as binge eating disorder. ${ }^{1-3}$ A slightly higher proportion of young women (13\%) meet criteria for the proposed DSM- 5 eating disorders. ${ }^{4}$ Eating disorders are marked by chronicity, relapse, distress, functional impairment, and risk for future obesity, depression, suicide attempts, anxiety disorders, substance abuse, and morbidity. ${ }^{5-9}$ As most individuals with eating disorders do not receive treatment and treatment is only effective for a subset of patients, an important public health priority is to develop and disseminate effective prevention programs. The goal of this review is to characterize the current knowledge regarding eating disorder prevention programs and offer thoughts on potentially fruitful directions for future research.

(C) 2013 by Wiley Periodicals, Inc.

*Correspondence to: Eric Stice, Oregon Research Institute, 1776 Millrace Drive, Eugene OR 97403. estice@ ori.org. 


\section{Efficacy Trials of Eating Disorder Prevention Programs}

Over 60 distinct eating disorder prevention programs have been evaluated in controlled trials. The first generation of prevention programs were largely psychoeducational in content and did not reduce eating disorder risk factors, eating disorder symptoms, or future eating disorder onset. ${ }^{10}$ The second generation of programs was also primarily didactic, but targeted empirically supposed risk factors, such as body dissatisfaction. Those programs produced significant reductions in select eating disorder risk factors, but rarely affected eating disorder symptoms or eating disorder onset. The third generation targeted empirically established risk factors, but did so with more interactive intervention content and often used persuasion principles from social psychology. These prevention programs tended to reduce eating disorder risk factors and eating disorder symptoms, sometimes reducing eating disorder onset. Meta-analytic reviews of published prevention trials indicated that significantly larger intervention effects tended to emerge for programs that target high-risk individuals (vs. a universal population), were interactive in nature, focused on risk factors shown to predict future onset of eating disorders (e.g., body dissatisfaction), and utilized a multisession format (vs. single-session). ${ }^{11}$

Efficacy trials have found that several eating disorder prevention programs have produced significant intervention effects on eating pathology. Efficacy trials aim to maximize internal validity and typically focus on homogeneous samples with little psychopathology, carefully standardize intervention implementation under tightly controlled settings, and involve research clinicians who are carefully trained and supervised. To our knowledge, only six prevention programs have significantly reduced eating disorder symptoms through at least 6month follow-up relative to assessment-only or educational brochure control conditions in trials, which have included universal prevention programs that target an entire population, ${ }^{12,13}$ selective prevention programs that target youth at high-risk for future onset of eating disorders, ${ }^{14,15}$ and indicated prevention programs that target individuals who have already shown onset of eating disorder symptoms. ${ }^{16}$ Effect sizes have tended to be small on average, but some programs have produced medium effect sizes for continuous outcomes, which we consider clinically significant.

Importantly, only two prevention programs have significantly reduced risk for future onset of eating disorders in trials. ${ }^{17,18}$ The first is a dissonance-based program in which young women critique the thin-ideal in a series of verbal, written, and behavioral exercises, which putatively causes cognitive dissonance and a consequent reduced subscription to the thinideal (the Body Project intervention). The second is an intervention that promotes participant-driven lasting improvements to dietary intake and physical activity with the goal of bringing participants into energy balance (the Healthy Weight intervention). Both programs have produced statistically significant and clinically meaningful $60 \%$ reductions in DSM-IV eating disorders over a 3-year follow-up relative to an assessment-only control condition, which corresponds to a medium effect size, but not relative to an expressive writing alternative intervention. ${ }^{17}$ A second-generation version of the Healthy Weight intervention also produced a significant and clinically meaningful $60 \%$ reduction in eating disorder onset over 2-year follow-up relative to an educational brochure control condition in an efficacy trial. ${ }^{18}$ One trial of an Internet-based eating disorder prevention program that include moderated discussions with a clinician found "no overall significant differences in onset of eating disorders between intervention and control groups," but reported that the intervention significantly reduced eating disorder onset over 3-year follow-up relative to control participants at one of the two sites involved in the trial. ${ }^{19}$ 


\section{Tests of the Intervention Theory of Eating Disorder Prevention Programs}

It is also important to provide rigorous tests of the intervention theory that underlies eating disorder prevention programs. For instance, a series of studies have provided support for the intervention theory for the Body Project. First, as hypothesized, reductions in thin-ideal internalization appear to mediate intervention effects on eating disorder symptom reductions. ${ }^{11,20}$ Second, the Body Project completely offset the risk for eating disorder onset conveyed by the most potent risk factor in a high-risk sample—denial of costs of pursuing the thin-ideal. ${ }^{21}$ Third, in support of the notion that dissonance induction drives the intervention effects, participants assigned to high-dissonance groups show greater reductions in eating disorder symptoms than those assigned to low-dissonance groups. ${ }^{22,23}$ Fourth, Stice and colleagues used fMRI to test whether the Body Project alters neural response to thin-ideal images and statements. Participants were randomized to the Body Project or an educational brochure control condition and completed pre- and post-fMRI scans in which they were exposed to images of models, images of average women, thin-ideal statements (e.g., narrow waist), and control descriptors (e.g., creative). A time $\times$ condition interaction indicated that Body Project participants showed a significantly greater pre-post reduction in activation of a key reward-valuation region (caudate) in response to thin-ideal statements (vs. control descriptors) relative to the slight increase observed in controls $(Z=3.24, p=$. $001, r=.67$, Fig. 1A). Body Project participants also showed a significantly greater pre-post reduction in activation of a key attention region (anterior cingulate cortex) in response to images of models (vs. average women) relative to the significant increase observed in controls $(Z=3.46, p<.001, r=.49$, Fig. 1B). Results provide objective biological evidence that the Body Project alters neural response to the ubiquitous thin-ideal stimuli that theoretically contribute to eating disorders, implying that after completing the Body Project, youth may not perceive the thin-ideal as representing a desirable goal and may automatically allocate less attention to the thin-ideal. Few studies have investigated the underlying theory behind other eating disorder prevention programs, which represents an important lacuna in the literature.

\section{Effectiveness Trials for Eating Disorder Prevention Programs}

A number of effectiveness trials have been conducted to date, although these have focused on a limited range of prevention programs. Effectiveness trials have greater external validity by investigating whether or not effects from highly controlled trials are maintained under increasingly "real world" conditions (e.g., use of community providers, practical levels of training, reliance on sustainable funding resources vs. research grants, partnerships with relevant communities, and samples with greater heterogeneity). One early trial study conducted by Matusek et al., ${ }^{24}$ in which college health educators were responsible for intervention delivery, but not participant recruitment, found that a dissonance-based eating disorder prevention program produced significant reductions in risk factors and eating disorder symptoms. Another effectiveness trial found that the Body Project produced significant reductions in risk factors, eating disorder symptoms, and impairment relative to educational brochure controls through 2- and 3-year follow-up when high school clinicians recruited students and delivered the program. ${ }^{2,25}$ Although this effectiveness trial found that the reductions in eating disorder symptoms persisted for 3-years, versus 2-years in the efficacy trial, ${ }^{17}$ the reduction in eating disorder onset did not reach significance, apparently due to the fact that the effectiveness sample was at lower-risk for eating disorder onset compared to the sample from the efficacy trial. In general, effect sizes from effectiveness trials have been small to moderate, though some effects are medium in magnitude.

Becker and colleagues conducted effectiveness trials demonstrating that peer-leaders in a sorority context could effectively deliver the Body Project when the intervention was 
tailored to their needs (e.g., provision of increased scripting in the program manual) and they received sufficient training. Importantly, these trials also showed that the Body Project retains its effectiveness even when it is semi-mandated and delivered in a universal format (i.e., all sorority members-both lower and higher risk participated). In the first study, new members to the local sororities of a small university were randomly assigned to receive the peer-led version of the Body Project or a media advocacy intervention. ${ }^{26}$ At 8-month follow-up, peer-led Body Project resulted in significantly greater reductions in thin-ideal internalization, body dissatisfaction, and maladaptive dieting compared to the media advocacy; participants in both conditions showed reductions in eating disorder symptoms.

The second study was a replication trial with a larger sample. ${ }^{27}$ Both the peer-led Body Project and the peer-led media advocacy intervention reduced eating disorder risk factors in the total sample at 8-month follow-up. Analyses of higher-and lower-risk participants, however, showed that whereas both interventions appeared to benefit higher-risk participants, only lower-risk participants who received the Body Project showed improvement.

In the third trial, Becker et al. ${ }^{28}$ examined whether the Healthy Weight intervention also could be sufficiently modified to allow peer-leaders to deliver this intervention. A greater degree of modification was needed to make the Healthy Weight program peer-leader friendly compared to the modification required for the Body Project. In this study, peer-led versions of Healthy Weight and the Body Project were compared. At post-intervention, the Body Project significantly decreased thin-ideal internalization, negative affect, and eating disorder symptoms relative to peer-led Healthy Weight. At 14 months, however, participants in both interventions showed reduced thin-ideal internalization, body dissatisfaction, negative affect, dietary restraint, and eating disorder symptoms. Within-group effect sizes showed that despite universal implementation within sororities, at 14-months, the twosession, peer-led version of the Body Project produced moderate to medium effect sizes, which were similar in magnitude to the 12-month effect sizes found with the three-session version in the large efficacy trial conducted by Stice et al. ${ }^{14}$ The positive effects for peer-led Body Project groups have replicated in an additional trial. ${ }^{29}$

At this point, it appears that the Body Project is the only eating disorder prevention program that warrants the APA's ${ }^{30}$ designation as an efficacious intervention, which means that it (a) has been shown to produce statistically significant and clinically meaningful effects when recruitment and delivery are conducted by natural providers (e.g., teachers, counselors, peer leaders etc.), as opposed to professional researchers, (b) has been found to significantly outperform active alternative prevention programs in multiple trials, and (c) has yielded positive effects for independent researchers.

\section{Dissemination of Eating Disorder Prevention Programs}

As the field of eating disorder prevention continues to make progress in identifying programs that yield positive effects in efficacy and effectiveness trials, it will be important to devote more attention to dissemination of evidence-based programs. The treatment literature has clearly shown, not just for eating disorders but also other forms of psychopathology, that many efficacious interventions remain underutilized in clinical practice, ${ }^{31-34}$ and there is little reason to suspect that the eating disorder prevention field will avoid this research-practice gap.

As suggested elsewhere, ${ }^{35}$ community participatory research may facilitate dissemination of efficacious programs. Piran ${ }^{36}$ pioneered the use of participatory research in eating disorder prevention research. Community participatory research involves engaging communities as 
equal partners in the research process with the intention of sharing power and decision making so as to enhance problem solving and integrate healthcare knowledge. The use of community participatory core principles ${ }^{37}$ facilitated dissemination of the Body Project to over 90 university campuses in partnership with a large national sorority (i.e., Tri Delta).

The Tri Delta dissemination effort also offers several lessons. First, although meta-analytic results suggest that programs that are delivered on a selective basis yield larger effect sizes, ${ }^{11}$ many communities (e.g., sororities, athletics) prefer to implement on a universal basis, and adoption of a participatory approach suggests that it may be helpful to respect such preferences. Further, in the case of the Body Project, it appears that universal administration within specific communities may not reduce effect sizes. Nonetheless, even if universal administration does reduce effect sizes of efficacious programs to some degree, it may be an acceptable price to pay for broad dissemination. As Kazdin and Blasé $e^{38}$ note, greater overall impact in large scale suffering may be achieved by having a smaller per person effect with a very large number of individuals versus a large effect with only a few individuals. Thus, we contend that researchers should continue to study both selective and universal administration of efficacious programs in dissemination efforts.

Second, it is clear that broad dissemination of efficacious programs will require infrastructure to train community providers and share implementation strategies. To date, in the case of the Body Project, a large national sorority provided the infrastructure. Although this was a positive first step, relying on just a few specific communities to disseminate to other communities is unrealistic. For instance, our sorority partner was much more interested in disseminating at the university level than high school, and high school dissemination of the Body Project thus has lagged even though empirical support exists for both age groups. As such, we suggest that the eating disorder prevention field must either be prepared to build its own infrastructure or to partner with prevention interventionists interested in other public health problems to share such an infrastructure.

Third, true scalability will require models for training trainers, not just infrastructure for training providers. ${ }^{39}$ To have a broad impact, efficacious prevention programs will need to be delivered to thousands of individuals. It is unrealistic to think that a small group of trainers will be sufficient to train the number of providers needed to accomplish this goal. Preliminary work in this area suggests that it is possible to train trainers for peer-led Body Project groups, ${ }^{29}$ but more research is needed.

Lastly, it will be important to consider the social context when attempting to broadly disseminate evidence-based prevention programs. Although the prevention programs with the strongest evidence base target body image concerns, this risk factor may be less germane for certain social groups, such as African-American women, who typically do not report the same degree of body image concerns as other ethnic groups. It will therefore be important to assess whether prevention programs are similarly effective for various ethnic groups and for populations that differ systematically from those studied in efficacy and effectiveness trials (e.g., lower socioeconomic groups). In this context, it should be noted that the Body Project has been found to be effective for Latina, Asian-American, and European-American young women, ${ }^{40}$ though research has not tested whether it is equally effective for African-

American young women, primarily because most trials have contained a limited representation of this population.

\section{Future Directions for Eating Disorder Prevention Research}

Although significant strides have been made over the last decade with regard to the development of efficacious and effective eating disorder prevention programs, there are 
several important directions for future research. First, prevention scientists should strive to develop interventions that produce larger effects and that show greater persistence, as there is room for improvement with even the most efficacious eating disorder prevention program. It may be fruitful to think outside the box with regard to the content of the prevention programs, such as making greater use of persuasion principles and social influence research from social psychology. Recent research implicates peer dieting in college as a prospective risk factor for eating disorder behaviors and attitudes 10 years later. ${ }^{41}$ Piran et al. ${ }^{42}$ also asserted that prevention efforts might be more sustainable if programming engages the social systems in which individuals are embedded. As such, it may be fruitful to expand existing peer-leader models as well as efforts to engage communities as partners in prevention efforts. Researchers can also attempt to improve session attendance and participation, facilitator competence, and fidelity of intervention delivery to test whether these factors contribute to larger intervention effects. In addition, we think it important to continue to try to develop efficacious prevention programs for preadolescent/early adolescent youth and universal prevention programs that are both efficacious and effective. ${ }^{43,44}$

Second, only a handful of efficacy trials have been designed to detect possible reductions in actual onset of eating disorders. Now that the field has made significant progress in reducing eating disorder risk factors in numerous trials, we should raise the bar and begin routinely testing for true prevention effects in large-scale efficacy trials. Although there are numerous challenges to doing so (e.g., need for longer follow-up periods, blinded interview assessment), major efficacy trials should be held to this standard.

Third, to date the majority of eating disorder prevention programs have largely targeted body image. Body dissatisfaction is a potent risk factor and also a useful target for prevention efforts because programs that reduce body dissatisfaction and prevent eating disorders can benefit multiple populations of individuals (i.e. those with body image concerns who would nonetheless not go on to develop an eating disorder as well as those for whom the eating disorder is prevented). Yet, there also are other risk factors for eating disorders (e.g., negative affect) and researchers should begin to evaluate whether targeting additional risk factors can improve the yield of eating disorder prevention programs. Moreover, based on our experience disseminating dissonance prevention, it has become clear that different communities have different desires when it comes to programming. For instance, athletics seems to prefer a modified version of the Healthy Weight program versus the Body Project. ${ }^{45}$ Creating a menu of efficacious programs will likely facilitate dissemination.

Fourth, as new programs are developed and successfully tested in efficacy trials, it will be vital to continue to conduct effectiveness trials that confirm that these interventions are effective when delivered by real-world community providers under ecologically valid conditions. Effectiveness trials conducted to date have revealed that interventions that have produced effects in tightly controlled efficacy trials in which research staff deliver the intervention often, though not always, produce smaller intervention effects under real-world conditions. On a related note, future research should also therefore seek to determine how best to maximize the effectiveness of prevention programs when delivered by local providers (e.g., improve training and supervision). More generally, it will be important for future effectiveness trials to be methodologically rigorous, such as using a randomized design with an active credible control condition, blinded diagnostic interviews to assess change in eating disorder symptoms and eating disorder onset, fidelity checks, validated measures, and long-term follow-up. The use of credible alternative control conditions is vital to reduce the possibility that demand characteristics inherent to randomized trials are driving the apparent intervention effects. This is particularly important because there are no 
objective biological measures of eating disordered behaviors, such as binge eating and compensatory behaviors (akin to objective doubly labeled water measures of total energy expenditure and intake). It would also be useful to begin to evaluate the effectiveness of the most promising eating disorder prevention programs in comparative trials, as this should provide clearer direction regarding which empirically supported prevention programs should be widely disseminated.

Fifth, it will be important to conduct systematic research on mediators that account for intervention effects (e.g., a reduction in thin-ideal internalization) and on the mechanism of action for prevention programs (e.g., dissonance induction) that are specified by the intervention theory for a particular prevention program. Such research should inform the design of even more effective refinements of extant programs or spark the design of new prevention programs that are more efficacious. Such studies might also reveal that a prevention program produces effects for entirely different reasons than those posited by the intervention theory.

Sixth, future studies should conduct research on the optimal methods for disseminating prevention programs with the strongest evidence-base. Although efficacious and effective eating disorder prevention programs exist, almost no research has been conducted on how to disseminate information about these programs and how to implement them broadly. More generally, one challenge involves obtaining "buy-in" from those who choose to adopt, or learn and implement the program. It is essential that we begin to conduct studies that elucidate barriers to successful dissemination of the most promising interventions. Research should also explore novel vehicles for implementing efficacious eating disorder prevention programs broadly and on a cost-effective basis, such as use of the Internet. ${ }^{16}$ Researchers should also consider dissemination strategies that target subpopulations at elevated risk for eating disorders, prioritizing social justice over cost-effectiveness.

Seventh, researchers should evaluate widely disseminated but untested programs. For example, DOVE has widely disseminated a body image improvement program and to our knowledge there is only one randomized controlled trial, which did not yield particularly impressive effects. ${ }^{46}$ Many lay people mistakenly believe that body image programs by definition prevent eating disorders, and this is clearly not the case. As such, eating disorder prevention researchers should be prepared to test programs developed by others (including those with more modest claims in terms of body image improvement) when they are widely marketed.

Finally, it would be useful to develop prevention programs that affect multiple public health problems, such as eating disorders and obesity, which often share risk factors. ${ }^{47}$ Prospective studies have found that body dissatisfaction, reported caloric restriction, and negative affect increase risk for escalation in eating disorder symptoms and body mass index (BMI) scores ${ }^{48,49}$ and onset of eating disorders and obesity, ${ }^{50-52}$ suggesting that some eating disorder risk factors increase risk for a variety of public health problems. Theoretically, elevated body dissatisfaction promotes both acute caloric deprivation dieting, that increase risk for excessive intake of energy dense foods, as well as negative affect, that prompts some individuals to consume energy dense foods as a means of improving affect. ${ }^{53}$ The Healthy Weight intervention has significantly reduced future increases in both eating disorder symptoms and body mass, and has significantly reduced future onset of eating disorders and obesity. ${ }^{18,54}$ An Internet version of the Body Project (eBody Project) has likewise reduced eating disorder symptoms relative to educational brochure and educational video control conditions from pre to post ( $\mathrm{M} d=.69$ ) and from pre to 1-year follow-up ( $\mathrm{M} d=.38$ ), and it has significantly reduced increases in BMI relative to both control conditions through 1-year follow-up ( $\mathrm{M} d=.82) .{ }^{55}$ Given that eating pathology increases the risk for onset of obesity, 
depression, and substance abuse, ${ }^{56}$ research should evaluate potential secondary benefits of eating disorder prevention programs on depression and substance abuse. Collaborating on dissemination of eating disorder prevention programs with prevention scientists targeting other health problems, such as substance use and risky sexual behavior, may likewise lead to strategic alliances with other advocacy communities that could circumvent competition for scarce resources (e.g., classroom time for universal interventions).

\section{Conclusion}

Substantial strides have been made in the development and evaluation of efficacious and effective eating disorder prevention programs over the last two decades. However, there is still considerable room for improvement in terms of the magnitude and duration of intervention effect. Perhaps most importantly, it is now time to intensively study methods of broadly disseminating evidence-based prevention programs so that we can realize the dream of reducing the population incidence of eating disorders.

\section{Acknowledgments}

Supported by MH001708, MH/DK061957, MH070699, DK080760, MH092468, and MH094448.

\section{References}

1. Hudson JI, Hiripi E, Pope HG Jr, Kessler RC. The prevalence and correlates of eating disorders in the National Comorbidity Survey replication. Biol Psychiatry. 2007; 61:348-358. [PubMed: 16815322]

2. Stice E, Marti CN, Shaw H, Jaconis M. An 8-year longitudinal study of the natural history of threshold, subthreshold, and partial eating disorders from a community sample of adolescents. J Abnormal Psychol. 2009; 118:587-597.

3. Wade TD, Bergin JL, Tiggemann M, Bulik CM, Fairburn CG. Prevalence and long-term course of lifetime eating disorders in an adult Australian twin cohort. Aust N Z J Psychiatry. 2006; 40:121128. [PubMed: 16476129]

4. Stice E, Marti N, Rohde P. Prevalence, incidence, impairment, and course of the proposed DSM-5 eating disorder diagnoses in an 8-year prospective community study of young women. J Abnormal Psychol. in press.

5. Crow SJ, Peterson CB, Swanson SA, Raymond NC, Specker S, Eckert ED, et al. Increased mortality in bulimia nervosa and other eating disorders. Am J Psychiatry. 2009; 166:1342-1346. [PubMed: 19833789]

6. Mond J, Hay P, Rodgers B, Owen C, Crosby R, Mitchell J. Use of extreme weight control behaviors with and without binge eating in a community sample: Implications for the classification of bulimictype eating disorders. Int J Eat Disord. 2006; 39:294-302. [PubMed: 16528679]

7. Le Grange D, Binford RB, Peterson CB, Crow SJ, Crosby RD, Klein MH, et al. DSM-IV threshold versus subthreshold bulimia nervosa. Int J Eat Disord. 2006; 39:462-467. [PubMed: 16715488]

8. Schmidt U, Andiappan M, Grover M, Robinson S, Perkins S, Dugmore O, et al. Randomised controlled trial of CD-ROM-based cognitive-behavioural self-care for bulimia nervosa. Br J Psychiatry. 2008; 193:493-500. [PubMed: 19043154]

9. Swanson SA, Crow SJ, Le Grange D, Swendsen J, Merikangas KR. Prevalence and correlates of eating disorders in adolescents: Results from the national comorbidity survey replication adolescent supplement. Arch Gen Psychiatry. 2011; 68:714-723. [PubMed: 21383252]

10. Pearson J, Goldklang D, Striegel-Moore RH. Prevention of eating disorders: Challenges and opportunities. Int J Eat Disord. 2002; 31:233-239. [PubMed: 11920984]

11. Stice E, Shaw H, Marti CN. A meta-analytic review of eating disorder prevention programs: Encouraging findings. Ann Rev Clin Psychol. 2007; 3:207-231. [PubMed: 17716054]

12. Neumark-Sztainer D, Butler R, Palti H. Eating disturbances among adolescent girls: Evaluation of a school-based primary prevention program. J Nutr Educ. 1995; 27:24-31. 
13. Stewart DA, Carter JC, Drinkwater J, Hainsworth J, Fairburn CG. Modification of eating attitudes and behavior in adolescent girls: A controlled study. Int J Eat Disord. 2001; 29:107-118. [PubMed: 11429973]

14. Stice E, Shaw H, Burton E, Wade E. Dissonance and healthy weight eating disorder prevention programs: A randomized efficacy trial. J Consult Clin Psychol. 2006; 74:263-275. [PubMed: 16649871]

15. Stice E, Rohde P, Gau J, Shaw H. An effectiveness trial of a dissonance-based eating disorder prevention program for high-risk adolescent girls. J Consult Clin Psychol. 2009; 77:825-834. [PubMed: 19803563]

16. Jones M, Luce K, Osborne M, Taylor K, Cunning D, Doyle A, et al. Randomized, controlled trial of an Internet-facilitated intervention for reducing binge eating and overweight in adolescents. Pediatrics. 2008; 121:453-462. [PubMed: 18310192]

17. Stice E, Marti CN, Spoor S, Presnell K, Shaw H. Dissonance and healthy weight eating disorder prevention programs: Long-term effects from a randomized efficacy trial. J Consult Clin Psychol. 2008; 76:329-340. [PubMed: 18377128]

18. Stice E, Rohde P, Shaw H, Marti N. Efficacy trial of a selected prevention program targeting both eating disorder symptoms and unhealthy weight gain among female college students. J Consult Clin Psychol. 2012; 80:164-170. [PubMed: 22122289]

19. Taylor C, Bryson S, Luce K, Cunning D, Celio A, Abascal L, et al. Prevention of eating disorders in at-risk college-age women. Arch Gen Psychiatry. 2006; 63:881-888. [PubMed: 16894064]

20. Seidel A, Presnell K, Rosenfield D. Mediators in the dissonance eating disorder prevention program. Behav Res Therapy. 2009; 47:645-653.

21. Stice E, Rohde P, Gau J, Shaw H. Effect of a dissonance-based prevention program on risk for eating disorder onset in the context of eating disorder risk factors. Prev Sci. 2012; 13:129-139. [PubMed: 21975593]

22. Green M, Scott N, Diyankova I, Gasser C, Pederson E. Eating disorder prevention: An experimental comparison of high level dissonance, low level dissonance, and no-treatment control. Eat Disord: J Treat Prev. 2005; 13:157-169.

23. McMillan W, Stice E, Rohde P. High- and low-level dissonance-based eating disorder prevention programs with young women with body image concerns: An experimental trial. J Consult Clin Psychol. 2011; 79:129-134. [PubMed: 21261438]

24. Matusek JA, Wendt SJ, Wiseman CV. Dissonance thin-ideal and didactic healthy behavior eating disorder prevention programs: Results from a controlled trial. Int J Eat Disord. 2004; 36:376-388. [PubMed: 15558649]

25. Stice E, Marti CN, Durant S. Risk factors for onset of eating disorders: Evidence of multiple risk pathways from an 8-year prospective study. Behav Res Therapy. 2011; 49:622-627.

26. Becker CB, Smith LM, Ciao AC. Peer facilitated eating disorders prevention: A randomized effectiveness trial of cognitive dissonance and media advocacy. J Counsel Psychol. 2006; 53:550 555.

27. Becker CB, Bull S, Smith LM, Ciao AC. Effects of being a peer-leader in an eating disorders prevention program: Can we further reduce eating disorder risk factors. Eat Disord: J Treat Prev. 2008; 16:444-459.

28. Becker CB, Wilson C, Williams A, Kelly M, McDaniel L, Elmquist J. Peer-facilitated cognitive dissonance versus healthy weight eating disorders prevention: A randomized comparison. Body Image. 2010; 7:280-288. [PubMed: 20638351]

29. Perez M, Becker CB, Ramirez A. Transportability of an empirically supported dissonance-based prevention program for eating disorders. Body Image: Int J Res. 2010; 7:179-186.

30. American Psychological Association Task Force on Psychological Intervention Guidelines. Template for Developing Guidelines: Interventions for Mental Disorders and Psychological Aspects of Physical Disorders. Washington, DC: American Psychological Association; 1995.

31. Becker CB, Zayfert C, Anderson E. Survey of psychologists' use and attitudes towards exposure therapy for PTSD. Behav Res Therapy. 2004; 42:277-292.

32. Freiheit SR, Vye C, Swan R, Cady M. Cognitive-behavioral therapy for anxiety: Is dissemination working? The Behav Therapist. 2004; 27:25-32. 
33. Mussell M, Crosby R, Crow S, Knopke A, Peterson C, Wonderlich S, et al. Utilization of empirically supported psychotherapy treatments for individuals with eating disorders: A survey of psychologists. Int J Eat Disord. 2000; 27:230-237. [PubMed: 10657896]

34. Rosen HJ, Pace-Savitsky K, Perry RJ, Kramer JH, Miller BL, Levenson RW. Recognition of emotion in the frontal and temporal variants of frontotemporal dementia. Dementia Geriatric Cogn Disord. 2004; 17:277-281.

35. Becker CB, Stice E, Shaw H, Woda S. Use of empirically supported interventions for psychopathology: Can the participatory approach move us beyond the research-to-practice gap? Behav Res Therapy. 2009; 47:265-274.

36. Piran, N. Re-inhabiting the body from the inside out: girls transform their school environment. In: Toman, DL.; Brydon-Miller, M., editors. From Subjects to Subjectivities: A Handbook of Interpretive and Participatory Methods. New York: New York University Press; 2001. p. 218-238.

37. Israel, BA.; Eng, E.; Schultz, AJ.; Parker, EA. Methods in Community-Based Participatory Research for Health. San Francisco: Jossey-Bass Publishers; 2005.

38. Kazdin AE, Blase SL. Rebooting psychotherapy research and practice to reduce the burden of mental illness. Perspect Psychol Sci (Sage Publications Inc). 2011; 6:21-37.

39. Wilson G, Zandberg LJ. Cognitive-behavioral guided self-help for eating disorders: Effectiveness and scalability. Clin Psychol Rev. 2012; 32:343-357. [PubMed: 22504491]

40. Rodriguez R, Marchand E, Ng J, Stice E. Effects of a cognitive-dissonance-based eating disorder prevention program are similar for Asian American, Hispanic, and White participants. Int $\mathrm{J}$ Eat Disord. 2008; 41:618-625. [PubMed: 18528871]

41. Keel PK, Forney KJ, Brown TA, Heatherton TF. Influence of college peers on disordered eating in women and men at 10-year follow-up. J Abnormal Psychol. in press.

42. Piran, N.; Levine, MP.; Steiner-Adair, C. Preventing Eating Disorders: A Handbook of Interventions and Special Challenges. Philadelphia, PA: Brunner/Mazel; 1999.

43. Levine, MP.; Smolak, L. The Prevention of Eating Problems and Eating Disorders: Theory, Research, and Practice. Mahwah, NJ: Lawrence Erlbaum Associates; 2006.

44. Wilksch SM, Wade TD. An investigation of temperament endophenotype candidates for early emergence of the core cognitive component of eating disorders. Psychol Med. 2009; 39:811-821. [PubMed: 18752731]

45. Becker CB, McDaniel L, Bull S, Powell M, McIntyre K. Can we reduce eating disorder risk factors in female college athletes? A randomized exploratory investigation of two peer-led interventions. Body Image. 2012; 9:31-42. [PubMed: 22019502]

46. Richardson SM, Paxton SJ, Thomson JS. Is BodyThink an efficacious body image and self-esteem program? A controlled evaluation with adolescents. Body Image. 2009; 6:75-82. [PubMed: 19188101]

47. Neumark-Sztainer D, Wall MM, Haines JI, Story MT, Sherwood NE, van den Berg PA. Shared risk and protective factors for overweight and disordered eating in adolescents. Am J Prev Med. 2007; 33:359-369. [PubMed: 17950400]

48. Haines J, Neumark-Sztainer D, Thiel L. Addressing weight-related issues in an elementary school: What do students, parents, and school staff recommend? Eat Disord: J Treat Prev. 2007; 15:5-21.

49. Johnson F, Wardle J. Dietary restraint, body dissatisfaction, and psychological distress: A prospective analysis. J Abnormal Psychol. 2005; 114:119-125.

50. Fairburn CG, Cooper Z, Doll HA, Davies BA. Identifying dieters who will develop an eating disorder: A prospective, population-based study. Am J Psychiatry. 2005; 162:2249-2255. [PubMed: 16330587]

51. Richardson LP, Davis R, Poulton R, McCauley E, Moffitt TE, Caspi A, et al. A longitudinal evaluation of adolescent depression and adult obesity. Arch Pediatrics Adolesc Med. 2003; 157:739-745.

52. Stice E, Presnell K, Shaw H, Rohde P. Psychological and behavioral risk factors for obesity onset in adolescent girls: A prospective study. J Consult Clin Psychol. 2005; 73:195-202. [PubMed: 15796626]

53. Stice E. A prospective test of the dual-pathway model of bulimic pathology: Mediating effects of dieting and negative affect. J Abnormal Psychol. 2001; 110:124-135. 
54. Stice E, Marti CN, Shaw H, O'Neil K. General and program-specific moderators of two eating disorder prevention programs. Int J Eat Disord. 2008; 41:611-617. [PubMed: 18528875]

55. Stice E, Rohde P, Durant S, Shaw H. A prototype Internet dissonance-based eating disorder prevention program for young women with body image concerns. J Consult Clin Psychol. 2012; 80:907-916. [PubMed: 22506791]

56. Stice E, Hayward C, Cameron R, Killen JD, Taylor CB. Body image and eating related factors predict onset of depression in female adolescents: A longitudinal study. J Abnorm Psychol. 2000; 109:438-444. [PubMed: 11016113] 
A

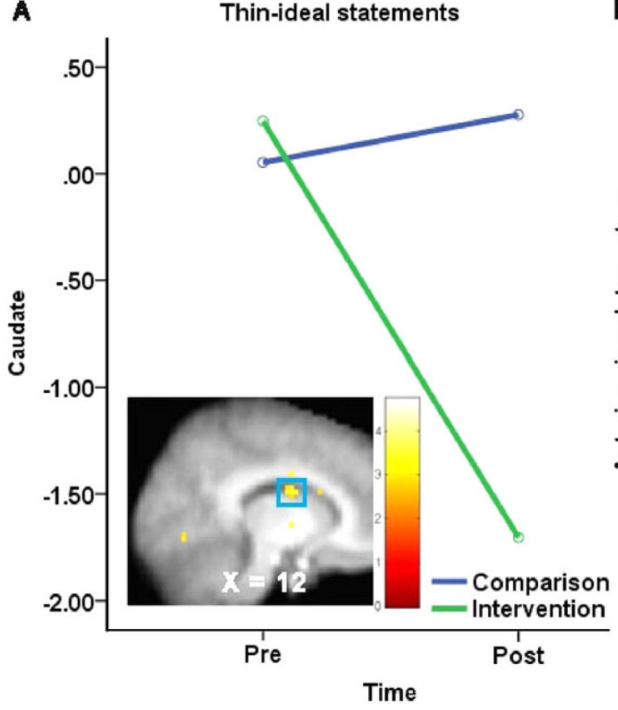

B

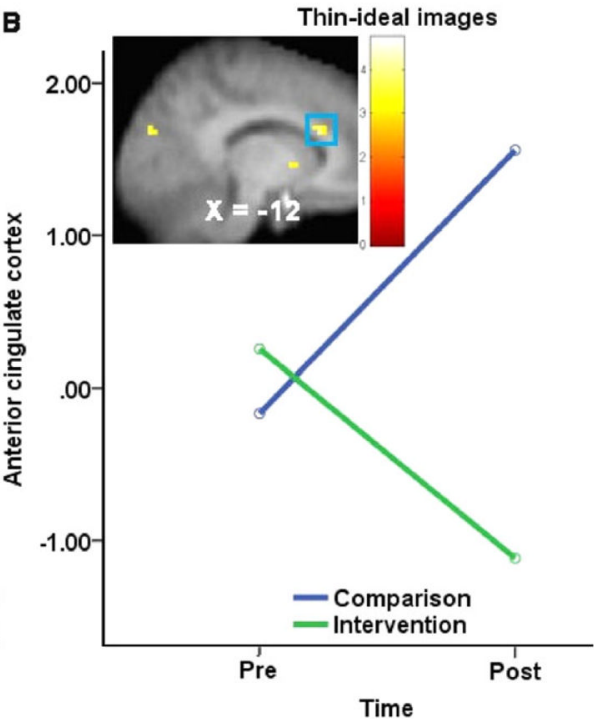

FIGURE 1.

Reduced activation in (A) the caudate and (B) the anterior cingulate cortex from pre to post for Body Project participants $(n=8)$ versus change observed for brochure controls $(n=7)$ during exposure to thin ideal images and statements. 